\title{
Efficient Time/Frequency Permutation of MIMO-OFDM Systems through Independent and Correlated Nakagami Fading Channels
}

\author{
Khodr A. SAAIFAN, Emad K. AL-HUSSAINI \\ Department of Electronics and Communications, Cairo University, Giza, Egypt \\ E-mail:khedrs@hotmail.com,emadh@eng.cu.edu.eg \\ Received August 16, 2009; revised September 28, 2009; accepted November 4, 2009
}

\begin{abstract}
Space-Time Frequency (STF) codes for MIMO-OFDM over block-fading channel can achieve rate $M_{t}$ and full-diversity $M_{t} M_{r} M_{b} L$ which is the product of the number of transmit antennas $M_{t}$, receive antennas $M_{r}$, fading blocks $M_{b}$ and channel taps $L$. In this article, time permutation is proposed to provide independent block-fading over Jake's Doppler power spectrum channel. Moreover, we show the performance variations of STF code as channel delay spread changes. Therefore, we introduce a frequency/time permutation technique in order to remove the frequency correlation among sub-carriers, which subsequently increases the coding gain and achieves maximum diversity. Finally, the symbol error rate (SER) performance of the proposed time/frequency permuted STF codes over independent and correlated MIMO antenna branches under Nakagami fading channel is simulated. We show that the proposed systems provide better performance and more robust to large values of antennas correlation coefficients in comparison with the un-interleaved one.
\end{abstract}

Keywords: MIMO, OFDM, Space-Time Frequency Coding, Nakagami Fading Channel, Time/Frequency Interleaving

\section{Introduction}

Achieving high data rate, full diversity gain and higher network capacity becomes the major requirements of wireless system providers. MIMO-OFDM system is one of the most attractive techniques to provide these capabilities.

Recently, some attention has been devoted to design STF codes for MIMO-OFDM system with $M_{t}$ transmit antennas, $M_{r}$ receive antennas, and $N$-OFDM tones through $L$ multi-path fading channel. There are several papers, which discussed the code structure to provide full diversity gain and high data rate. In [1], W. Su et al. proposed the design of full diversity space frequency block code (SFBC) with rate-1 for any number of transmit antennas and arbitrary power delay profiles. The rate- $M_{t}$ full diversity SFBC was proposed in [2] for any arbitrary number of transmit antennas. However, because a zeropadding matrix has to be used when $N$ is not an integer multiple of $M_{t} L$, the symbol transmission rate $M_{t}$ cannot be always guaranteed.

In [3], better diversity gains through block-fading channels can be obtained, that was done by spreading the coding across multiple fading blocks. In [4], they studied the error performance results of STF codes in MIMOOFDM systems for a variety of system configurations and channel conditions. The maximum diversity is the product of time diversity, frequency diversity and space diversity as shown in [5]. Recently in [6], W. Zhang et al. proposed a systematic design of high-rate STF codes for MIMO frequency-selective block-fading channels. By spreading the algebraic coded symbols across different OFDM sub-channels, transmit antennas and fading blocks, the proposed STF codes can achieve a rate- $M_{t}$ and a full diversity of $M_{t} M_{r} M_{b} L$, where $M_{b}$ is the number of independent fading blocks in the code-words. To achieve the full-diversity performance of STF code, maximumlikelihood (ML) decoding must be employed. In order to decrease the large complexity of ML decoding, sphere decoder can be considered to achieve near-ML performance $[7,8]$. For block-fading channels, the performance of STF-coded OFDM is much better than SF coding as demonstrated in [9].

In MIMO-OFDM systems, the DFT operation introduces correlation into the channel frequency response at different sub-carriers $[10,11]$, making its performance var- 
ies as the delays between paths vary.

The outline of the paper is as follows. Section 2 describes the channel statistics and system model. The suggested time/frequency permutations of high rate STF codes structure proposed in [6] for independent and correlated Nakagami fading are introduced in Section 3. In Section 4, we provide simulation results for the performance of the proposed scheme. Finally, some conclusions are made in Section 5.

\section{Channel Statistics and System Models}

Before investigating permutation schemes for MIMOOFDM systems equipped with $M_{t}$ transmit antennas, $M_{r}$ receive antennas in mobile radio channels, we briefly describe the channel statistics, emphasizing the separation property of mobile wireless channels, which is crucial for simplifying our time/frequency permutation. In this section we also briefly describe a MIMO-OFDM system.

\subsection{Statistics of Mobile Radio Channels}

The channels between each pair of transmit and receive antennas are assumed to have $L$ independent delay paths and the same power delay profile. The channel impulse response between $m_{t}{ }^{\text {th }}$ transmit antenna and $m_{r}{ }^{\text {th }}$ receive antenna can be modeled as

$$
h_{m_{t}, m_{r}}(t ; \tau)=\sum_{l=0}^{L-1} \alpha_{m_{t}, m_{r}}^{l}(t) \delta\left(t-\tau_{l}\right)
$$

where $\tau_{l}$ is the delay of the $l^{\text {th }}$ path, and $\alpha_{m_{t}, m_{r}}^{l}(t)$ is complex amplitude of the $l^{\text {th }}$ path between $m_{t}{ }^{\text {th }}$ transmit antenna and $m_{r}{ }^{\text {th }}$ receive antenna. $\alpha_{m_{t}, m_{r}}^{l}(t)$ 's are modeled as a complex random fading signals with Nakagarni-m distributed fading amplitudes and uniform phases. Nakagami m-distribution fading model [12] is one of the most versatile, in the sense that it has greater flexibility and accuracy in matching some experimental data than Rayleigh, log-normal, or Rician distributions. The Rayleigh distribution is a special case when the fading parameter $m=1$. It can approximate Rice distribution for $m>1$. Moreover, it is assumed that all path gains between any pair of transmit and receive antennas follow the same power profile, i.e., $E\left[\left|\alpha_{m_{t}, m_{r}}^{l}(t)\right|^{2}\right]=\sigma_{l}^{2}>0$ for any given $\left(m_{t}, m_{r}, l\right)$. The powers of the paths are normalized such that $\sum_{l=0}^{L-1} \sigma_{l}^{2}=1$. Using Equation (1), the frequency responses of the time-varying radio channel at time $t$ is

$$
H_{m_{t}, m_{r}}(t, f)=\sum_{l=0}^{L-1} \alpha_{m_{t}, m_{r}}^{l}(t) \exp \left(-j 2 \pi f \tau_{l}\right)
$$

The MIMO channel is assumed to be spatially correlated for any $\left(m_{t}, m_{r}\right)$, where $m_{t}=1, \ldots M_{t}, m_{r}=1, \ldots M_{r}$, and independent for any $l$ where, $l=0, \ldots L-1$. Let $\rho_{m_{t}, m_{t}^{\prime}}^{T X}$ denotes the spatial correlation coefficient between $\alpha_{m_{t}, m_{t}}(t)$ and $\alpha_{m_{t}^{\prime}, m_{r}}^{l}(t)$ defined as

$$
\rho_{m_{t}, m_{t}^{\prime}}^{T x}=\left\langle\alpha_{m_{t}, m_{r}}^{l}(t), \alpha_{m_{t}^{\prime}, m_{r}}^{l}(t)\right\rangle
$$

The spatial correlation coefficient observed at the receiver has also been extensively studied in the literature and is given as

$$
\rho_{m_{r}, m_{r}^{\prime}}^{R x}=\left\langle\alpha_{m_{t}, m_{r}}^{l}(t), \alpha_{m_{t}, m_{r}^{\prime}}^{l}(t)\right\rangle
$$

Given Equations (3) and (4), the symmetrical correlation matrices at transmitter and the receiver can be defined respectively as

$$
\mathbf{R}_{T x}=\left[\begin{array}{cccc}
\rho_{11}^{T x} & \rho_{12}^{T x} & \cdots & \rho_{1 M_{t}}^{T x} \\
\rho_{21}^{T x} & \rho_{22}^{T x} & \cdots & \rho_{2 M_{t}}^{T x} \\
\vdots & \vdots & \ddots & \vdots \\
\rho_{M_{t} 1}^{T x} & \rho_{M_{t} 2}^{T x} & \cdots & \rho_{M_{t} M_{t}}^{T x}
\end{array}\right]_{M_{t} \times M_{t}}
$$

and,

$$
\mathbf{R}_{R x}=\left[\begin{array}{cccc}
\rho_{11}^{R x} & \rho_{12}^{R x} & \cdots & \rho_{1 M_{r}}^{R x} \\
\rho_{21}^{R x} & \rho_{22}^{R x} & \cdots & \rho_{2 M_{r}}^{R x} \\
\vdots & \vdots & \ddots & \vdots \\
\rho_{M_{r} 1}^{R x} & \rho_{M_{r} 2}^{R x} & \cdots & \rho_{M_{r} M_{r}}^{R x}
\end{array}\right]_{M_{r} \times M_{r}}
$$

The spatial correlation matrix $\mathbf{R}$ of the MIMO radio channel is the Kronecker product of the spatial correlation matrix at the transmitter and the receiver and is given by [13]

$$
\mathbf{R}=\mathbf{R}_{T x} \otimes \mathbf{R}_{R x}
$$

where $\otimes$ denotes the Kronecker product.

The correlation function of the frequency response for different times and frequencies is

$$
\begin{array}{r}
\Phi_{m_{t}, m_{t}^{\prime}}(\Delta t, \Delta f)=E\left[H_{m_{t}, m_{r}}^{*}(t, f) H_{m_{t}^{\prime}, m_{r}}(t+\Delta t, f+\Delta f)\right] \\
=\sum_{l=0}^{L-1} E\left[\alpha_{m_{t}, m_{r}}^{l^{*}}(t) \alpha_{m_{t}^{\prime}, m_{r}}^{l}(t+\Delta t)\right] \exp \left(-j 2 \pi \Delta f \tau_{l}\right)
\end{array}
$$

Assume Jake's Doppler power spectrum [14], therefore the correlation of the $l^{\text {th }}$ path is given by

$$
E\left[\alpha_{m_{t}, m_{r}}^{l^{*}}(t) \alpha_{m_{t}^{\prime}, m_{r}}^{l}(t+\Delta t)\right]=\rho_{m_{t}, m_{t}^{\prime}}^{T x} \sigma_{l}^{2} J_{0}\left(2 \pi f_{D} \Delta t\right)
$$

where $\sigma_{l}^{2}$ represents the power of $l^{\text {th }}$ path, $f_{D}$ is the Doppler frequency, and $J_{\mathrm{O}}(x)$ is the zero order Bessel function of the first kind. Substitute Equation (9) in Equation (8), then Equation (8) can be rewritten as 


$$
\begin{aligned}
& \Phi_{m_{t}, m_{t}^{\prime}}(\Delta t, \Delta f) \\
& =\rho_{m_{t}, m_{t}^{\prime}}^{T x} J_{0}\left(2 \pi f_{D} \Delta t\right) \sum_{l=0}^{L-1} \sigma_{l}^{2} \exp \left(-j 2 \pi \Delta f \tau_{l}\right) \\
& =\rho_{m_{t}, m_{t}^{\prime}}^{T x} \Phi_{t}(\Delta t) \Phi_{f}(\Delta f)
\end{aligned}
$$

where $\Phi_{t}(\Delta t)$ is the time domain correlation function and $\Phi_{f}(\Delta f)$ is the frequency domain correlation function. From Equation (10), the time-frequency domain channel correlation function of $H_{m t, m r}(t, f)$ can be separated as the product of the spatial correlation coefficient, the time domain channel correlation, and the frequency domain channel correlation, which are dependent on the antenna separation, the Doppler frequency, and multi-path delay spread respectively.

For an OFDM system with block length $\boldsymbol{T}$ and tone spacing (sub-channel spacing) $\Delta f=1 / T$, the correlation function for different blocks and tones can be written as

$$
\Phi_{m_{t}, m_{t}^{\prime}}(\Delta t, \Delta f)=\rho_{m_{t} m_{t}^{\prime}}^{T x} \Phi_{t}(k T) \Phi_{f}(n / T)
$$

\subsection{MIMO-OFDM System Model}

Consider a STF-coded MIMO-OFDM system with $M_{t}$ transmit antennas, $M_{r}$ receive antennas and $N$ sub-carriers operating over a frequency-selective multi-path fading channel. The MIMO-OFDM system with code permutations considered in this paper is shown in Figure 1.

The source $\mathbf{S}$ generates $N_{s}=N M_{t} M_{b}$ information symbols from the discrete alphabet $\boldsymbol{A}$, which are quadrature amplitude modulation (QAM) normalized into the unit power. Using a mapping $f: \mathbf{S} \rightarrow \mathbf{C}$, an information symbol vector $\mathbf{S} \in A^{N s}$ is parsed into blocks and mapped onto a STF codeword to be transmitted over the $M_{t}$ transmit antennas and $M_{b}$ OFDM blocks. Each STF codeword C can be expressed as a $N \times M_{b} M_{t}$ matrix.

$$
\mathbf{C}=\left[\begin{array}{llll}
\mathbf{C}^{1} & \mathbf{C}^{2} & \cdots & \mathbf{C}^{M_{b}}
\end{array}\right]
$$

where the $N \times M_{t}$ matrix $\quad \mathbf{C}^{m_{b}}=\left[\begin{array}{llll}\mathbf{c}_{1}^{m_{b}} & \mathbf{c}_{2}^{m_{b}} & \cdots & \mathbf{c}_{M_{t}}^{m_{b}}\end{array}\right]$ for $m_{b}=1, \ldots M_{b}$ denotes the sub-codeword ready to be sent during the time epoch $m_{b}$. The $m_{t}^{\text {th }}\left(m_{t}=1, \ldots M_{t}\right)$ column of

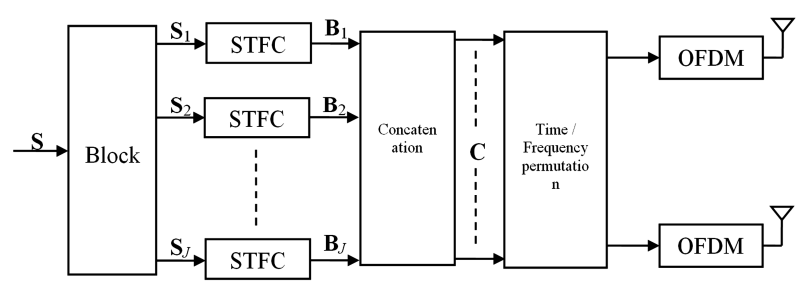

Figure 1. MIMO-OFDM system with code permutation to combat channel correlation.
$\mathbf{C}^{m b}$ denoted by $\mathbf{c}_{m_{t}}^{m_{b}}$ is sent to the OFDM block at the $m_{t}^{\text {th }}$ transmit antenna during the time epoch $m_{b}$. After inverse fast Fourier transform (IFFT) modulation and cyclic prefix (CP) insertion, OFDM symbols are sent from all transmit antennas simultaneously.

At the receiver, after matched filtering, removing the cyclic prefix, and applying FFT, the received signal at the received signal at the $m_{r}^{\text {th }}$ receive antenna during the time epoch $m_{b}$ is given by

$$
\mathbf{Y}_{m_{r}}^{m_{b}}=\sqrt{\frac{\rho}{M_{t}}} \sum_{m_{t}=1}^{M_{t}} \operatorname{diag}\left(\mathbf{c}_{m_{t}}^{m_{b}}\right) \mathbf{H}_{m_{t}, m_{r}}^{m_{b}}+\mathbf{Z}_{m_{r}}^{m_{b}}
$$

where

$$
\mathbf{H}_{m_{t}, m_{r}}^{m_{b}}=\left[\begin{array}{llllll}
H_{m_{t}, m_{r}}^{m_{b}}(0) & H_{m_{t}, m_{r}}^{m_{b}}(1) & \cdots & H_{m_{t}, m_{r}}^{m_{b}}(N-1)
\end{array}\right]^{T}
$$

is the $m_{b}^{\text {th }}$ OFDM block channel frequency response vector between $m_{t}^{\text {th }}$ transmit antenna and $m_{r}{ }^{\text {th }}$ receive antenna and $\mathbf{Z}_{m_{t}}^{m_{b}}$ denotes the complex discrete AWGN process with zero mean and unit variance at the $m_{r}^{\text {th }}$ receive antenna. The factor $\sqrt{\rho / M_{t}}$ in Equation (13) ensures that the average SNR at each receive antenna is independent on the number of transmit antennas.

\section{Time/Frequency Permuted STF Codes}

STF coding proposed in [6] can achieve rate of $M_{t}$ and full diversity for any number of transmit antennas and any arbitrary channel power delay profiles. It was constructed by applying the layering concept along with algebraic code components, which was introduced in the design of threaded algebraic space-time (TAST) code [15]. The STF code structure spreads the algebraic code components in adjacent sub-carriers and adjacent time slots that suffer from high correlation introduced by DFT operation and time correlation respectively. In this section, time/frequency permuted STF code structure is introduced into STF code structure of [6] in order to remove the effect of channel correlation among the code components and achieve better diversity order.

\subsection{STF Codes Structures}

Let $N_{p}=2^{\left\lceil\log _{2} L\right\rceil}, N_{q}=2^{\left\lceil\log _{2} M_{t}\right\rceil}$, and $K=N_{p} \cdot N_{q}$, then a block of $N_{s}$ transmitted information symbols $\mathrm{S}=\left[\mathrm{S}_{1}, \mathrm{~S}_{2}, \ldots S_{N M t M b}\right]^{T}$ are parsed into $J(J=N / K)$ equal size sub-blocks. Each sub-block $\mathbf{S}_{j} \in A K^{M t M b}(j=1,2 \ldots, J)$ is respectively encoded into an STF code matrix $\mathbf{B} j$ of size $K \times M_{t} M_{b}$ through the following steps:

1) Each subblock $\mathbf{S}_{j}(j=1,2 \ldots, J)$ are parsed into $N_{q}$ information vector $\mathbf{s}_{n_{q}} \in A^{N_{p} M_{t} M_{b}}\left(n_{q}=1,2, \cdots, N_{q}\right)$. 
2) Generate algebraic code sub-block $\overline{\mathbf{X}}_{n_{q}}$ by applying a fully-diverse unitary transformations $\boldsymbol{\Theta}$ into each information vector $\mathbf{s}_{n_{q}}\left(n_{q}=1,2, \cdots N_{q}\right)$ to generate $N_{q}$ threads by

$$
\begin{aligned}
& \overline{\mathbf{X}}_{n_{q}}=\left[\begin{array}{lllllll}
\overline{\mathbf{X}}_{n_{q}, 1}^{1} & \ldots & \overline{\mathbf{X}}_{n_{q}, N_{L}}^{1} & \ldots & \overline{\mathbf{X}}_{n_{q}, 1}^{M_{b}} & \ldots & \overline{\mathbf{X}}_{n_{q}, N_{L}}^{M_{b}}
\end{array}\right] \\
& =\left[\begin{array}{lllll}
X_{n_{q}}(1) & X_{n_{q}}(2) & \cdots & X_{n_{q}}(\bar{N})
\end{array}\right] \\
& =\boldsymbol{\Theta} \mathbf{s}_{n_{q}}
\end{aligned}
$$

where $\bar{N}=N_{p} M_{t} M_{b}$, and $\boldsymbol{\Theta}$ is the first principal $\bar{N} \times \bar{N}$ unitary matrix of the following matrix

$$
\boldsymbol{\Psi}=\mathbf{F}_{\bar{M}}^{H} \operatorname{diag}\left(1, \varphi, \cdots, \varphi^{\bar{M}-1}\right)
$$

where $\bar{M}=2^{\left\lceil\log _{2} \bar{N}\right\rceil}, \quad \mathbf{F}_{\bar{N}}^{H}$ is the $\bar{M} \times \bar{M}$ discrete Fourier transform (DFT) matrix, and $\varphi=\exp (j 2 \pi / 4 \bar{M})$.

3) Applying the layering concept to construct the encoder sub-matrices $\overline{\mathbf{X}}_{n_{p}}^{m_{b}}\left(n_{p}=1, \cdots N_{p}\right.$ and $\left.m_{b}=1, \cdots M_{b}\right)$.

$$
\begin{aligned}
& \overline{\mathbf{X}}_{n_{p}}^{m_{b}}=\left[\begin{array}{llll}
\overline{\mathbf{X}}_{1, n_{p}}^{m_{b} T} & \varphi \overline{\mathbf{X}}_{2, n_{p}}^{m_{b} T} & \cdots & \varphi^{N_{q}-1} \overline{\mathbf{X}}_{N_{q}, n_{p}}^{m_{b}}{ }^{T}
\end{array}\right] \\
& =\left(\begin{array}{cccc}
X_{1}\left(k_{n_{p}}^{m_{b}}+1\right) & \varphi X_{2}\left(k_{n_{p}}^{m_{b}}+1\right) & \cdots & \varphi^{N_{q}-1} X_{N_{q}}\left(k_{n_{p}}^{m_{b}}+1\right) \\
X_{1}\left(k_{n_{p}}^{m_{b}}+2\right) & \varphi X_{2}\left(k_{n_{p}}^{m_{b}}+2\right) & \cdots & \varphi^{N_{q}-1} X_{N_{q}}\left(k_{n_{p}}^{m_{b}}+2\right) \\
\vdots & \vdots & \ddots & \vdots \\
X_{1}\left(k_{n_{p}}^{m_{b}}+M_{t}\right) & \varphi X_{2}\left(k_{n_{p}}^{m_{b}}+M_{t}\right) & \cdots & \varphi^{N_{q}-1} X_{N_{q}}\left(k_{n_{p}}^{m_{b}}+M_{t}\right)
\end{array}\right)
\end{aligned}
$$

where $\phi=\varphi^{1 / N_{q}}$ and $k_{n_{p}}^{m_{b}}=\left(n_{p}-1\right) M_{t}+\left(m_{b}-1\right) N_{p} M_{t}$.

4) Re-arrange the elements of $\overline{\mathbf{X}}_{n_{p}}^{m_{b}}$ by $\overline{\mathbf{X}}_{n_{p}}^{m_{b}}\left(m_{t}^{\prime}, n_{q}^{\prime}\right)$ $=\overline{\mathbf{X}}_{m}^{u}\left(m_{t}, n_{q}\right): n_{q}^{\prime}=\left\{\left(m_{t}+n_{q}-2\right)_{\bmod M_{t}}+1\right\}$, and $m_{t}^{\prime}=\left\{m_{t}+\left\lceil\frac{n_{q}}{M_{t}}\right\rceil-1\right\}$, for $1 \leq m_{t}<M_{t}, 1 \leq n_{q} \leq N_{q}$

$$
\left(\begin{array}{cccc}
X_{1}\left(k_{n_{p}}^{m_{b}}+1\right) & \varphi X_{2}\left(k_{n_{p}}^{m_{b}}+1\right) & \cdots & \varphi^{M_{t}-1} X_{M_{t}}\left(k_{n_{p}}^{m_{b}}+1\right) \\
\varphi^{N_{q}-1} X_{N_{q}}\left(k_{n_{p}}^{m_{b}}+1\right) & X_{1}\left(k_{n_{p}}^{m_{b}}+2\right) & \cdots & \varphi^{M_{T}-2} X_{M_{t}-1}\left(k_{n_{p}}^{m_{b}}+2\right) \\
\vdots & \vdots & \ddots & \vdots \\
\varphi X_{2}\left(k_{n_{p}}^{m_{b}}+M_{t}\right) & \varphi^{2} X_{3}\left(k_{n_{p}}^{m_{b}}+M_{t}\right) & \cdots & \varphi^{N_{q}-1} X_{N_{q}}\left(k_{n_{p}}^{m_{b}}+M_{t}\right)
\end{array}\right)(17)
$$

then, the $K \times M_{t} M_{b}$ code matrix $\mathbf{B}_{i}$ is constructed as

$$
\mathbf{B}_{i}=\left(\begin{array}{cccc}
\overline{\mathbf{X}}_{1}^{1} & \overline{\mathbf{X}}_{1}^{2} & \cdots & \overline{\mathbf{X}}_{1}^{M_{b}} \\
\overline{\mathbf{X}}_{2}^{1} & \overline{\mathbf{X}}_{2}^{2} & \cdots & \overline{\mathbf{X}}_{2}^{M_{b}} \\
\vdots & \vdots & \ddots & \vdots \\
\overline{\mathbf{X}}_{N_{L}}^{1} & \overline{\mathbf{X}}_{N_{L}}^{2} & \cdots & \overline{\mathbf{X}}_{N_{L}}^{M_{b}}
\end{array}\right)
$$

The STF coding applies the same coding strategy to every sub-block $\mathbf{B}_{j}(j=1,2, \cdots, J)$, then the rate- $M_{t} \mathrm{STF}$ code $\mathbf{C} \in C^{N \times M_{t} M_{b}}$ is of the form

$$
\mathbf{C}=\left[\begin{array}{llll}
\mathbf{B}_{1}^{T} & \mathbf{B}_{2}^{T} & \cdots & \mathbf{B}_{J}^{T}
\end{array}\right]^{T}
$$

It is clear that, each thread of codeword $X_{n_{q}}\left(n_{\bar{n}}\right)$ $\left(n_{q}=1,2, \cdots N_{q}\right.$ and $\left.n_{\bar{n}}=1,2, \cdots \bar{N}\right)$ is spread over space, time and frequency dimensions. Therefore, the STF code structure is not optimum in spreading the code components of each thread on adjacent sub-carriers that suffer from high correlation introduced by DFT operation. However, if the power delay profile of the channel is available at the transmitter side, further improvement can be achieved by developing an interleaving strategy (can reduce the correlation between adjacent sub-carriers) which explicitly considers the power delay profile. In addition, since the STF code structure maintains its diversity gain from sending the OFDM blocks through independent fading blocks, we shall introduce time permutation to achieve independent fading blocks through MIMO channels that suffer from high correlation introduced by Doppler power spectrum.

\subsection{Time/Frequency Permutation Schemes}

The assumption of independent fading at the branches is acceptable if the antennas are spaced sufficiently apart with respect to the radio frequency (RF) carrier wavelength. In this case, $\rho_{m_{t} m_{t}^{\prime}}^{T X}=0, \forall m_{t} \neq m_{t}^{\prime}$, and $\rho_{m_{t} m_{t}^{\prime}}^{T X}=1, \forall m_{t}=m_{t}^{\prime}$,then Equation (11) will be reduced to the autocorrelation function [10]

$$
\Phi_{m_{t}, m_{t}}(k, n)=J_{0}\left(2 \pi f_{D} k T\right) * \sum_{l=0}^{L-1} \sigma_{l}^{2} \exp \left(-j 2 \pi \tau_{l} n / T\right)
$$

Obviously, the sources of channel correlation are caused by the time domain channel correlation, and the frequency domain channel correlation. Our objective is to find the separation parameters $k$ and $n$ for MIMOOFDM system which produce zero time and frequency correlations then permute the algebraic code components of $\mathbf{B}_{j}(j=1, \ldots J)$ at zero time frequency correlation to maximize the diversity gain.

$$
K_{c}=\min _{k}\left[J_{0}\left(2 \pi f_{D} k T\right)\right]
$$

$$
N_{c}=\min _{0 \leq n \leq N-1}\left[\sum_{l=0}^{L-1} \sigma_{l}^{2} \exp \left(-j 2 \pi \tau_{l} n / T\right)\right]
$$

The zeros of the Bessel functions (Equation (21)) play a dominant role in our applications. The Bessel functions have infinite number of zeros. The maxima and minima of $J_{0}$ steadily decrease in absolute value as $k$ increases. 
The first five zeros of $J_{0}$ are 2.4048, 5.5201, 8.6537, 11.7915, and 14.9309. The interval between the last two is 3.1394 , which is already close to $\pi$. The larger roots are approximately $(v-1 / 4) \pi$, where $v$ is the number of the root. To break the time correlation of the channel, verify independent fading block and realize high-rate full-diversity STC of [6], the $M_{b}$-OFDM blocks of STC matrix $\mathbf{B}_{j}(j=1, \ldots J)$ should be transmitted at time difference of $K_{c}=\left\lceil\frac{2.4048}{2 \pi f_{D} T}\right\rceil$. For large coherence time or equivalently low Doppler spread of the fading, high interleaving size is required to break the memory of the channel.

The optimum sub-carriers separation factor $N_{c}$ (see Equation (22)) can be easily found via low-complexity computer search. However, closed-form solutions for specific cases are reported in [1].

Based on the knowledge of channel separations factors $N_{c}$ and $K_{c}$, time/frequency permuted STF code can be introduced using the following steps:

1) Distribute the STC blocks over independent fading blocks by permuting the $u$-OFDM blocks of STC matrix $\mathbf{B}_{j}(j=1, \ldots J)$ with those blocks at time $u K_{c}$, $\left(u=2, \cdots M_{b}\right)$.

2) Apply frequency permutation into each pair of code matrices $\mathbf{B}_{j}$ and $\mathbf{B}_{j}$, where $j^{\prime}=j+N_{b}, N_{b}=N_{c} / K$, $j=\left[1, \cdots, N_{b}\right]+2\left(n_{b}-1\right) N_{b}$ and $n_{b}=1,2, \ldots, J / 2 N_{b}$ by permuting rows $K / 2+1, \cdots, K$ of $\mathbf{B}_{j}$ with the rows $1, \cdots, K / 2$ of $\mathbf{B}_{j}$.

3) Further permutation should be done to break the rest of channel frequency correlation by permuting each pair of rows $\left(n_{1}, n_{2}\right)$, where $n_{1}=2, \cdots K / 2$ and $n_{2}=K / 2+2, \cdots K$ for all code matrices $\mathbf{B}_{j}(j=1, \cdots J)$ with the corresponding pair of rows at block distances $u\left(M_{b}-1\right) K_{c}$ where $(u=2, \cdots, K / 2)$.

By performing the above steps as shown in Figure 2, the code components $X_{n_{q}}\left(n_{\bar{n}}\right) \quad\left(n_{q}=1,2, \cdots N_{q}\right.$ and $\left.n_{\bar{n}}=1,2, \cdots \bar{N}\right)$ of each thread of code matrix $\mathbf{B}_{j}$ are affected by independent fading blocks which subsequently achieve maximum diversity gain.

Examples of STF codes and permuted STF codes for $M_{t}=2, L=2$ are shown Figures 3 and 4 . For $M_{b}=1$, STF codes will be, in fact, the SF codes of [16]. The rate-2 SF code structure and the suggested time/frequency permutation (antenna 1 is shown only) are shown in Figure 3.

The rate- 2 STF code structure and the suggested time/frequency permutation for $M_{b}=2$ are shown in Figure 4.

\section{Simulation Results}

In this section, we simulated the proposed permutation

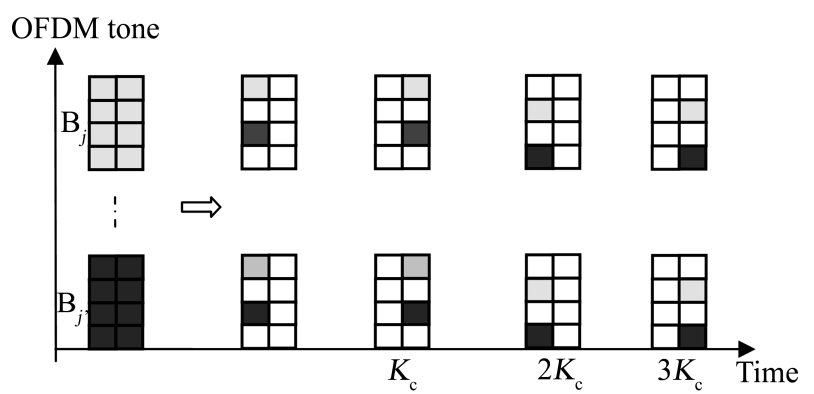

Figure 2. The suggested time/frequency permutation of STF codes.

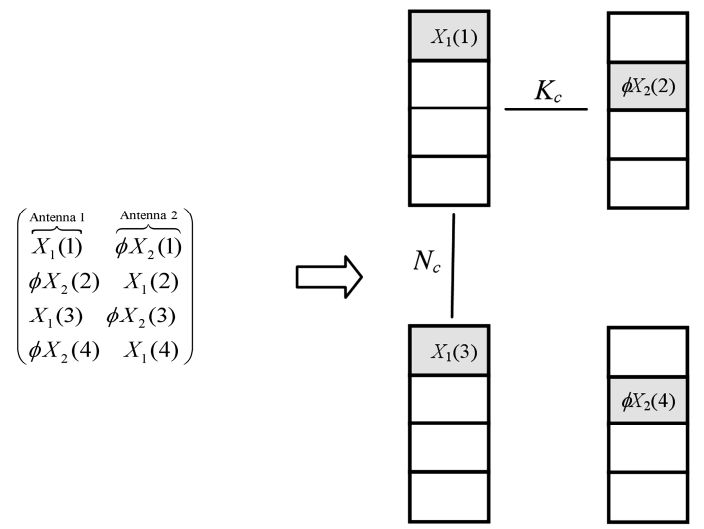

Figure 3. Rate-2 time/frequency permuted SF code (T/FP-SF).

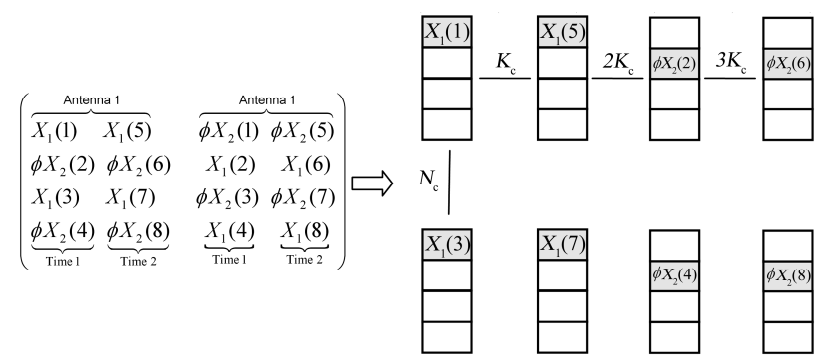

Figure 4. Rate-2 time/frequency permuted STF code (T/FP-STF).

scheme and compared with the non-permuted STF codes for different power delay profiles of the channel. We present average symbol-error rate (SER) curves as functions of the average SNR. Then we illustrate the performance of the proposed permutation for SF codes through correlated Nakagami fading channels. To investigate the performance of the proposed time/frequency permutation of STF codes over frequency-selective fading channels, we perform the simulation experiments and compare with the STF codes [6] for MIMO-OFDM systems. In the simulation, we use a $2 \times 2$ system with 128 OFDM tones and 4QAM transmission scheme, thus the spectral efficiency is $4 \mathrm{bit} / \mathrm{s} / \mathrm{Hz}$, ignoring the cyclic prefix. The bandwidth of OFDM system is $1 \mathrm{MHz}$ and the length of the cyclic prefix is 32 , i.e., $32 \mu \mathrm{s}$. Hence the duration of one OFDM symbol (cyclic prefix excluded) is $T=128 \mu \mathrm{s}$. A two-ray Nakagami fading channel statis- 
tics model is considered with the equal gain, Doppler spread $f_{D}=200 \mathrm{~Hz}$, and fading depth $m=0.5,1$ and 2 .

It is to be noted that $m=0.5$ represents the worst fading situation that can be represented by Nakagami distribution. This case can be countered in bad urban mobile radio. When $m=1$, we obtain Rayleigh fading channel. Finally, $m=2$ represents the best considered situation in which the fading is less than that of Rayleigh.

\subsection{Performance Comparison for Different Delay Spreads}

The first set of experiments is conducted to compare the performance of the proposed scheme with STF codes for different path delay of the two-ray model. A simple two-ray, equal-power delay profile, with a delay $\tau$ microseconds between the two rays is assumed. Simulation is carried out for two cases: 1) $8 \mu \mathrm{sec}$ (optimum permutation $N_{c}=8$ ) and 2) $20 \mu \mathrm{sec}$ (optimum permutation $N_{c}=16$ ). For Doppler spread $f_{D}=200 \mathrm{~Hz}$ the optimum time separation is 14 OFDM symbols to ensure independent fading blocks, therefore the interleaved STF code is spanned over 56 OFDM symbols.

Figures 5, 6 and 7 depict the improvement in SER performance offered by the proposed time/frequency permutations through independent Nakagami fading channel with different $m$. The values of the fading depth considered are $m=0.5,1$, and 2 respectively.
It can be observed from these figures that the SER performance of STF codes [6] varied as the delay spread of the channel changed. The SER performance of STF codes is further improved as delay spread of the channel increased. Such an improvement is attributed to the large coding gain induced by multi-path fading channels with a larger delay spread. The performance of the STF code degraded significantly from the $20 \mu \mathrm{s}$ case to the $8 \mu \mathrm{s}$ case, whereas the performance of the STF code using time/ frequency permutation was almost the same for the two delay profiles.

We can see that the T/FP-STF codes have better SER performance than the non-permuted STF codes. For $\tau=8 \mu \mathrm{s}$ case, there is an improvement of about $3.2 \mathrm{~dB}$ for $\mathrm{SF}$ codes and an improvement of about $1.8 \mathrm{~dB}$ for the STF codes at a SER of $10^{-4}$ when $m=1$. Therefore; the proposed interleaving method offering higher code gains making it more robust to small delay spread. This confirms that by careful interleaver design, the performance of the STF codes can be significantly improved.

From Table 1, it is clear that the SNR decreases with the increase of $m$. The performance of the interleaved codes is not sensitive to the variation in the channel time delay spread. In all of cases considered, the required SNR of the time/frequency interleaved codes is lower than that needed for the un-interleaved one to achieve the same SER.

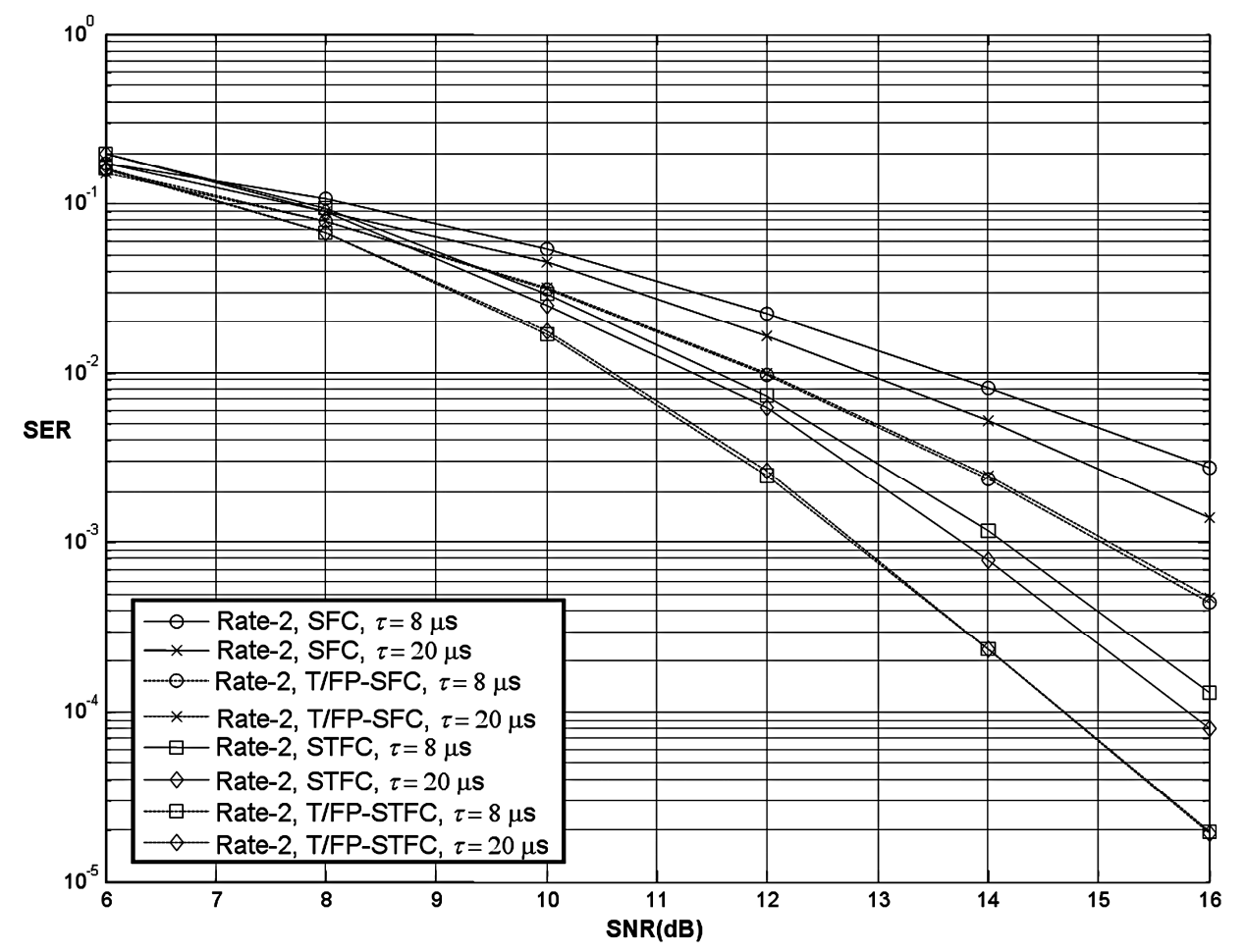

Figure 5. Average SER versus SNR of $2 \times 2$, MIMO-OFDM system through independent Nakagami fading channel $m=0.5$ with different delay spread. 


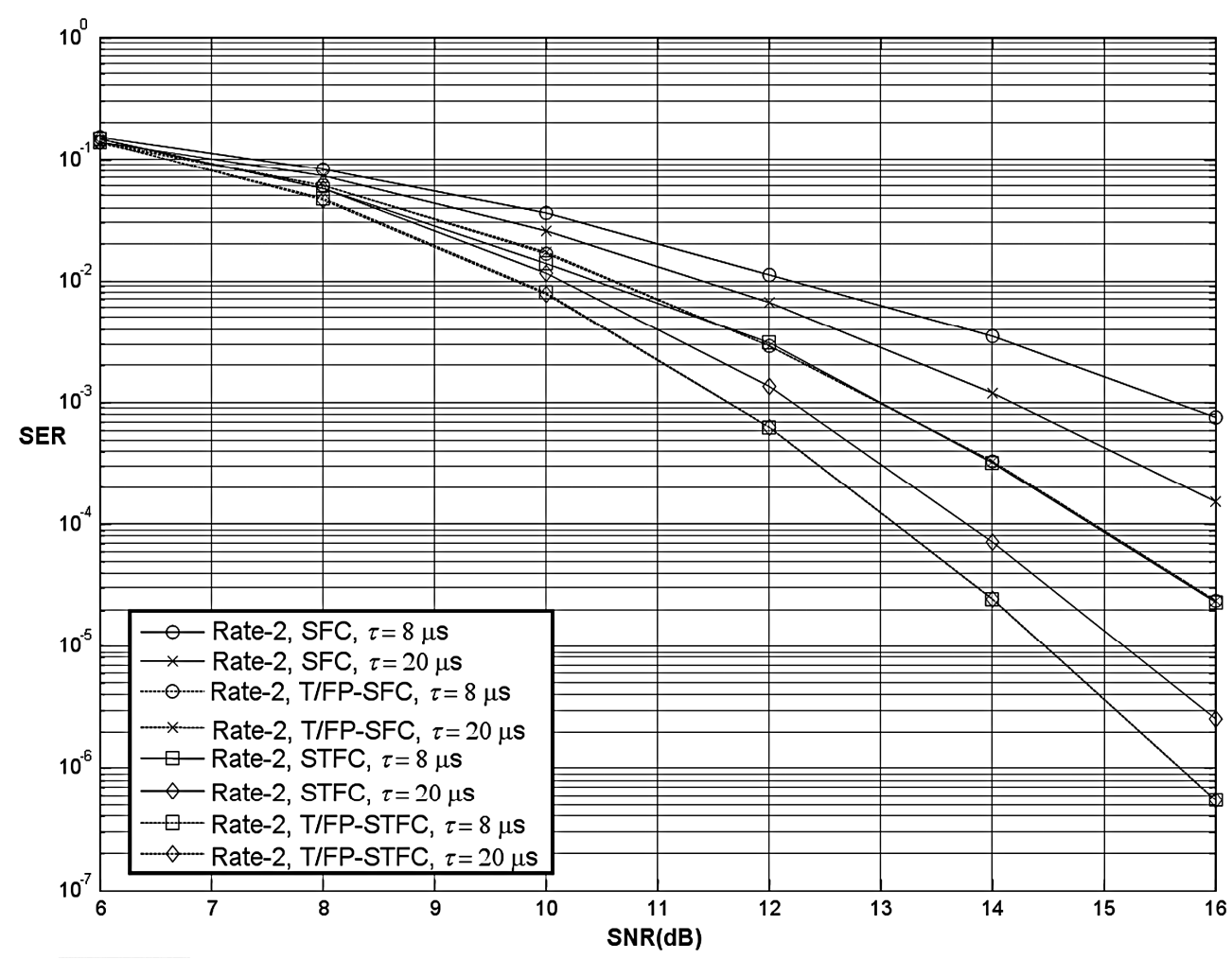

Figure 6. Average SER versus SNR of $2 \times 2$, MIMO-OFDM system through independent Nakagami fading channel $m=1$ with different delay spread.

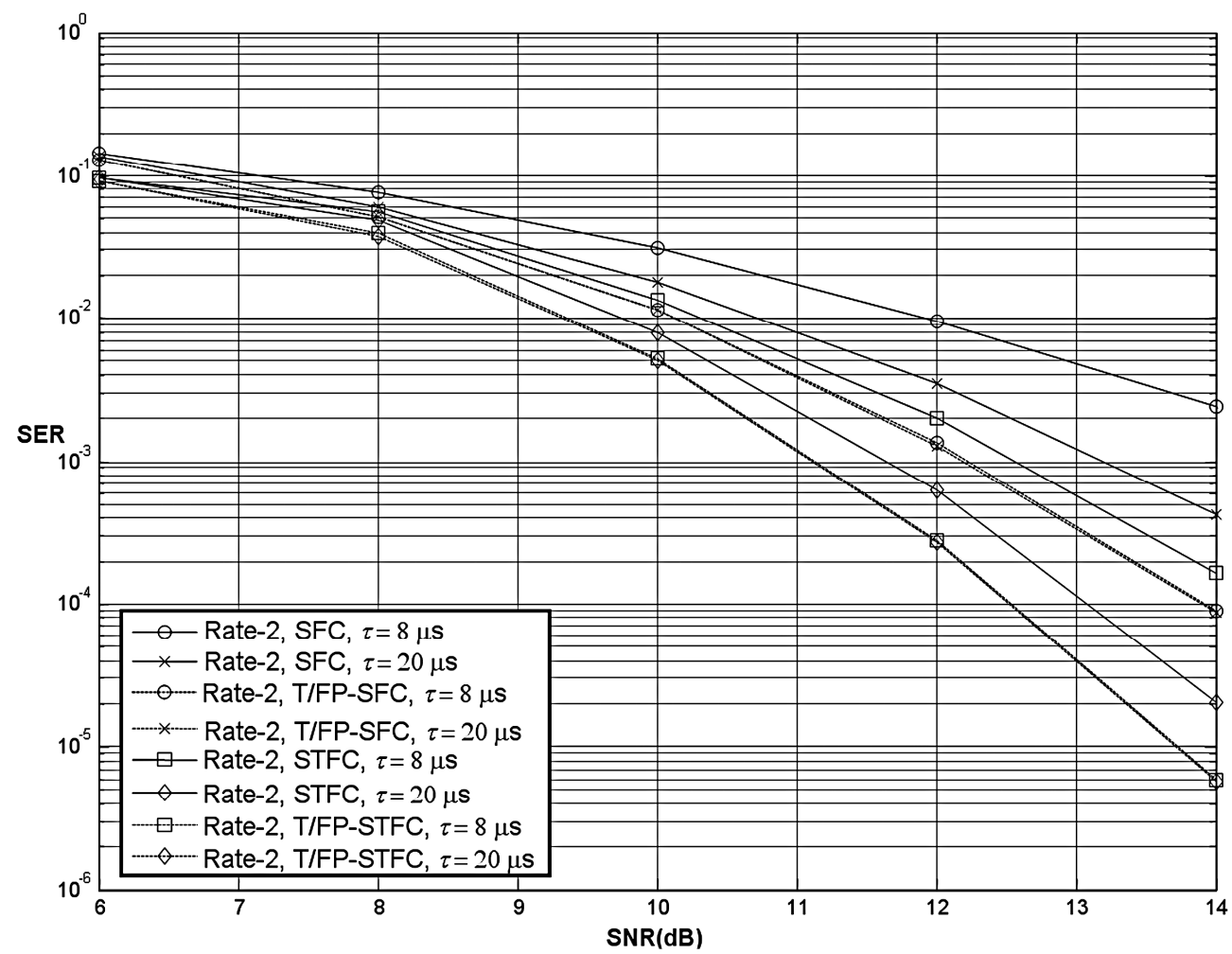

Figure 7. Average SER versus SNR of $2 \times 2$, MIMO-OFDM system through independent Nakagami fading channel $m=2$ with different delay spread. 
Table 1. SNR required to obtain a $\mathrm{SER}=10^{-4}$ for $\mathrm{STFC}$ and T/FP-STFC at different time delay spread.

\begin{tabular}{|c|c|c|c|c|c|c|}
\hline \multirow{2}{*}{ M } & \multicolumn{2}{|c|}{ SFC } & T/FP-SFC & \multicolumn{2}{|c|}{ STFC } & T/FP-STFC \\
\hline & $8 \mu \mathrm{sec}$ & $20 \mu \mathrm{sec}$ & $8 \mu \mathrm{sec} \quad 20 \mu \mathrm{sec}$ & $8 \mu \mathrm{sec}$ & $20 \mu \mathrm{sec}$ & $8 \mu \mathrm{sec} \quad 20 \mu \mathrm{sec}$ \\
\hline 0.5 & $20.8 \mathrm{~dB}$ & $19.4 \mathrm{~dB}$ & $18 \mathrm{~dB}$ & $16.3 \mathrm{~dB}$ & $15.8 \mathrm{~dB}$ & $14.7 \mathrm{~dB}$ \\
\hline 1 & $18.1 \mathrm{~dB}$ & $16.2 \mathrm{~dB}$ & $14.9 \mathrm{~dB}$ & $14.9 \mathrm{~dB}$ & $13.8 \mathrm{~dB}$ & $13.13 \mathrm{~dB}$ \\
\hline 2 & $17.4 \mathrm{~dB}$ & $15.2 \mathrm{~dB}$ & $13.9 \mathrm{~dB}$ & $14.3 \mathrm{~dB}$ & $13.1 \mathrm{~dB}$ & $12.53 \mathrm{~dB}$ \\
\hline
\end{tabular}

\subsection{Performance Comparisons over Correlated Nakagami Fading Channels}

MIMO system with closely spaced antenna elements is considered here. Our aim is to analyze the influence of the Nakagami-m fading parameter and the effect of antenna correlation on the SER performance of the rate- $2 \mathrm{SF}$ code, and the proposed T/FP-SF code depicted in Figure 3.

Figure 8 shows the SER degradation as the correlation coefficients between the transmitting antenna branches $\rho$ vary from 0 up to 0.8 . Similar correlation is assumed between receiving antenna branches. Simulation is carried out for two cases: 1) Transmitter correlated Nakagami MIMO fading channel case: $\mathbf{R}_{t}=\left[\begin{array}{ll}1 & \rho \\ \rho & 1\end{array}\right]$, and $\mathbf{R}_{r}=\left[\begin{array}{ll}1 & 0 \\ 0 & 1\end{array}\right]$, and 2) Doubly correlated Nakagami MIMO fading channel case: $\mathbf{R}_{t}=\left[\begin{array}{ll}1 & \rho \\ \rho & 1\end{array}\right], \quad$ and $\mathbf{R}_{r}=\left[\begin{array}{ll}1 & \rho \\ \rho & 1\end{array}\right]$. The values of the fading depth considered are $\mathrm{m}=0.5,1$, and 2 respectively. It is clear that the SER increases with the increase of correlation coefficient $\rho$. At $\rho=0$, the received signals are independent and the codes practically achieves full diversity reception gain. It is clear that the probability of error decreases with the increase of $m$, which is with the decrease of the severity of fading.

From these figures, it is clear that the systems under consideration appreciably dominate the systems considered in [6].

\section{Conclusions}

In this paper, the limitation for achieving full-diversity of STF-coded OFDM is introduced. The limitation arises due to the fact that the algebraic code components are spread in adjacent sub-carriers that suffer from high correlation introduced by DFT operation. Assuming that the power delay profile of the channel is available at the transmitter, we proposed an efficient time-frequency interleaving scheme to further improve the performance. Based on simulation results, we can draw the following conclusions.
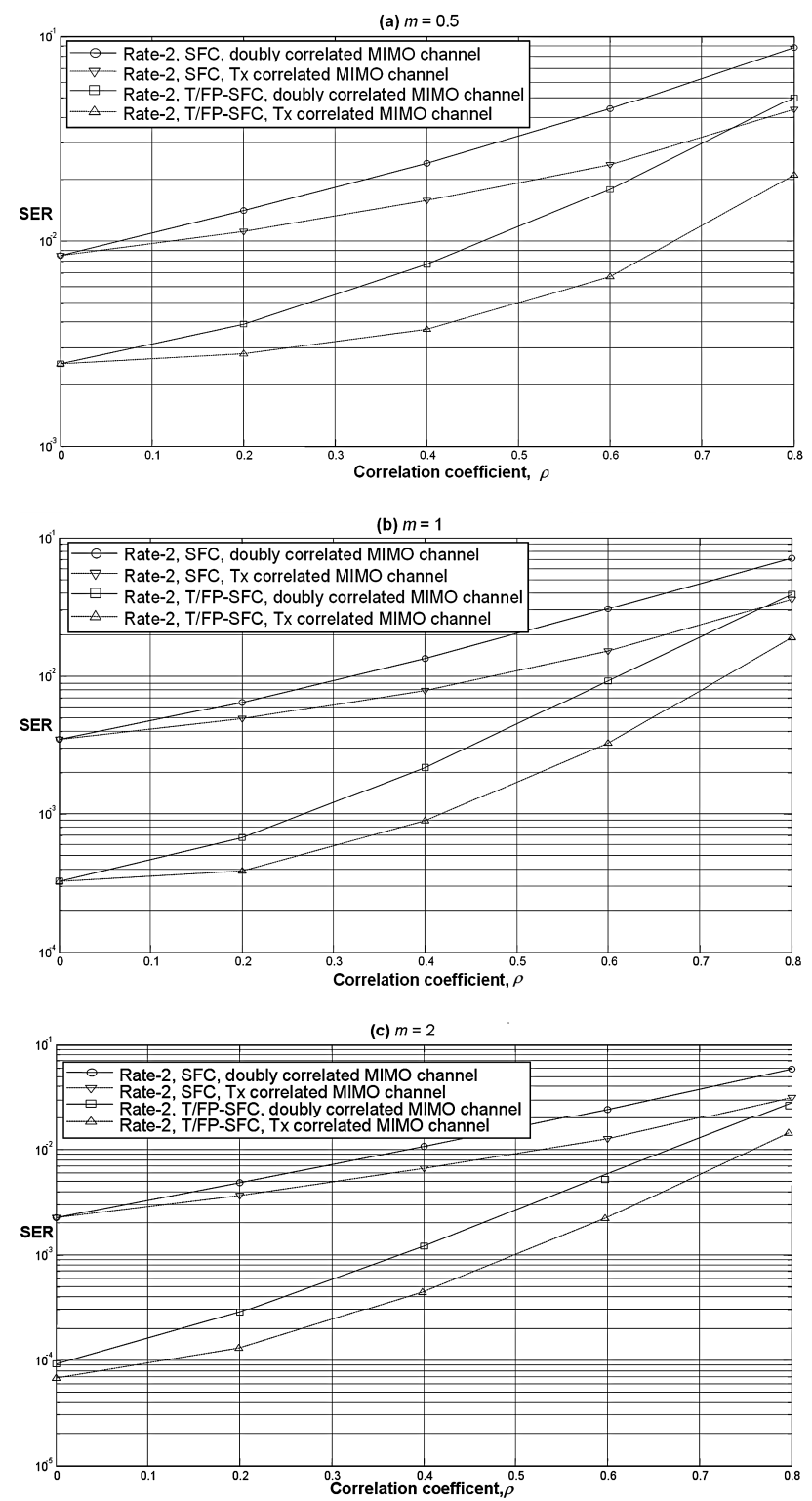

Figure 8. Average SER versus correlation coefficients for $2 \times 2$ MIMO-OFDM systems at $\mathrm{SNR}=14 \mathrm{~dB}$.

First, the proposed time/frequency permutations STF codes offer considerable performance improvement over previously reported results. Second, the applied interleaving scheme can have a significant effect on the overall performance of the STF code through correlated and independent Nakagami fading channels. 


\section{References}

[1] W. Su, Z. Safar, and K. J. R. Liu, "Full-rate full-diversity space: Frequency codes with optimum coding advantage," IEEE Transactions on Information Theory, Vol. 51, pp. 229-249, January 2005.

[2] T. Kiran and B. S. Rajan, "A systematic design of high-rate full-diversity space frequency codes for MIMO-OFDM systems," in Proceedings IEEE International Symposium Information Theory, pp. 2075-2079, September 2005.

[3] H. E. Gamal and A. R. Hammons Jr., "On the design of algebraic space-time codes for MIMO block fading channels," IEEE Transactions on Information Theory, Vol. 49, pp. 151-163, January 2003.

[4] M. Fozunbal, S. W. McLaughlin, and R. W. Schafer, "On space-time-frequency coding over MIMO-OFDM systems," IEEE Transactions on Wireless Communication, Vol. 4, pp. 320-331, January 2005.

[5] W. Su, Z. Safar, and K. J. R. Liu, "Towards maximum achievable diversity in space, time, and frequency: Performance analysis and code design," IEEE Transactions on Wireless Communication, Vol. 4, pp. 1847-1857, July 2005.

[6] W. Zhang, X. G. Xia, and P. C. Ching, "High-Rate fulldiversity space-time-frequency codes for broadband MIMO block fading channels," IEEE Transaction on Communication, Vol. 55, pp. 25-34, January 2007.

[7] E. Viterbo and J. Boutros, "A universal lattice code decoder for fading channels," IEEE Transactions on Information Theory, Vol. 45, No. 5, pp. 1639-1642, July 1999.

[8] M. O. Damen, A. Chkeif, and J. C. Belfiore, "Lattice code decoder for space-time codes," IEEE Communication Letters, Vol. 4, No. 5, pp. 161-163, May 2000.
[9] W. Zhang, X. G. Xia, and K. B. Letaief, "Space-time/ frequency coding for MIMO-OFDM in next generation broadband wireless systems," IEEE Wireless Communications Magazine, Vol. 14, No. 3, pp. 32-43, June 2007.

[10] Y. Li, L. J. Cimini, and N. R. Sollenberger, "Robust channel estimation for OFDM systems with rapid dispersive fading channels," IEEE Transactions on Communication, Vol. 46, No. 7, pp. 902-915, July 1998.

[11] X. Wang and K. J. R. Liu, "Channel estimation for multicarrier modulation systems using a time-frequency polynomial model," IEEE Transactions on Communication, Vol. 50, No. 7, pp. 1045-1048, July 2002.

[12] M. Nakagami, "The m-distribution: A general formula of intensity distribution of rapid fading", in W. C. Hoffman (ed.), Statistical Methods in Radio Wave Propagation, Pergamon Press, New York, pp. 3-36, 1960.

[13] W. C. Jakes (2nd), "Microwave mobile communications," IEEE Press, New York, 1994.

[14] K. I. Pedersen, J. B. Andersen, J. P. Kermoal, and P. E. Mogensen, "A stochastic multiple-input multiple-output radio channel model for evaluation of space-time coding algorithms," In Proceedings of Vehicular Technique Conference, pp. 893-897, September 2000.

[15] H. E. Gamal and M. O. Damen, "Universal space-time coding," IEEE Transactions on Information Theory, Vol. 49, pp. 1097-1119, May 2003.

[16] W. Zhang, X. G. Xia, P. C. Ching, and H. Wang, "Rate two full-diversity space-frequency code design for MIMOOFDM," Proceedings of IEEE Workshop Signal Process, Advanced Wireless Communications, New York, pp. 321-325, June 2005. 